\section{BDA LEADS CALL FOR PLASMA DONATIONS TO FIGHT COVID-19}

The British Dental Association (BDA) is urging dentists and dental team members in England who have tested positive for COVID-19 to put themselves forward as potential donors for the NHS convalescent plasma programme, currently being used in clinical trials to improve the recovery of people with the virus.

Leading the call is Birmingham dentist and newly elected BDA Chair, Eddie Crouch, who developed COVID-19 during lockdown, has fully recovered and has volunteered for the programme.

Baseline figures from a large-scale study at Birmingham University - covering over 1,500 dental team members across the Midlands - showed $16 \%$ of dentists, hygienists, therapists and nurses had COVID-19 antibodies prior to the resumption of face to face care on 8 June using enhanced PPE and new safety protocols. This compared to between $6-10 \%$ for the general population in the region.

Donated plasma is being transfused into people who are still unwell, and the antibodies could save lives, with the

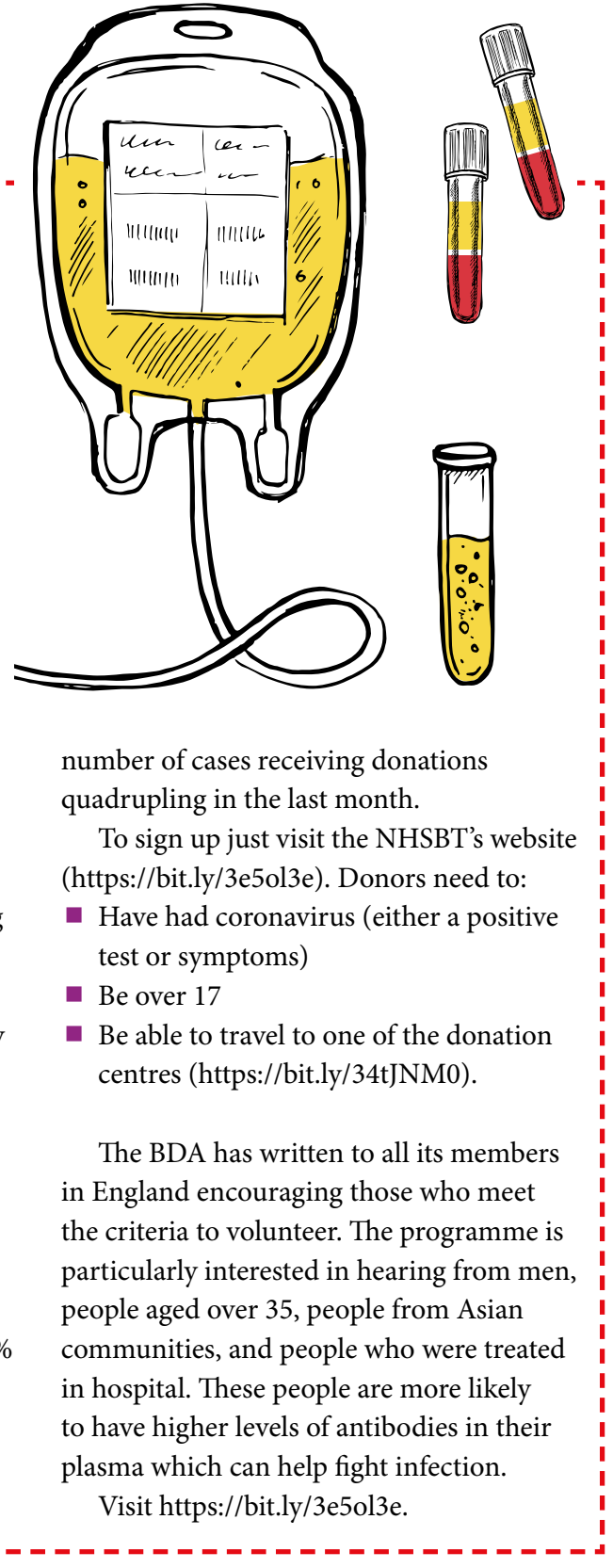

Dental murse salary survey 2020

The British Association of Dental Nurses (BADN) has launched its 2020 Salary Survey.

The Survey is open to all dental nurses, non-members as well as BADN members, to find out how much dental nurses in the UK are being paid, as well as whether they were paid during lockdown. The Survey will provide data to support the BADN's campaign for recognition of dental nurses' contribution to oral healthcare in the UK, and for dental nurses working in general dental practice to be paid NHS rates, instead of minimum wage.

BADN invites all dental nurses, but particularly those working in practice, to complete the survey - which is anonymous. Dental nurses completing the Survey will need their P60 for the tax year ending April 2020 - all employees should have received this in April 2020 from their employer.

The Survey can be accessed from the BADN website www.badn.org.uk or via https://cutt.ly/SalarySurvey2020. The closing date is 31 December 2020.

\title{
BSDHT AND BADT LAUNCH CONSULTATION TO CHANGE LEGISLATION
}

The British Society of Dental Hygiene and Therapy (BSDHT) and the British Association of Dental Therapists (BADT) have been working on the NHS England project to change legislation and in collaboration with the Scottish, Welsh and Northern Ireland Governments, are now consulting the public on proposed changes to the Human Medicines Regulations 2012 to include the use by dental hygienists and therapists of a mechanism called exemptions.

Dental hygienists and dental therapists are trained, indemnified and competent in the use of certain prescription only medicines. The mechanisms by which they use these medicines are varied and currently they can administer, sell and supply only by a patient specific direction (PSD) or a patient group directive (PGD). These mechanisms have advantages and disadvantages and the profession have decided to unite to find a more effective solution to providing these medicines in a timely and cost-efficient manner.

Being able to use the exemptions mechanism will mean that:

- Dental hygienists and dental therapists will be able to supply and administer certain medicines to patients that would normally need a prescription from a dentist

- Dentists would be relieved of an administration burden

- Fewer patients would be turned away for treatment due to changes in their needs

- The dental hygienist or dental therapist would be able to treat their patient with the medicines they are trained, indemnified and competent to use in a timely fashion, resulting in fewer appointments with fewer costs incurred by patients and dental practices

- There is a reduction in the number of interruptions to a dentist to ask them to prescribe a medicine.

This would help dentists, dental hygienists and dental therapists to provide safer, quicker and more comfortable care for their patients.

The Public Consultation can be found at https://www.england.nhs.uk/medicines-2/ chief-professions-officers-medicinesmechanisms-programme/ and runs from 15 October to 10 December 2020.

Links to engagement events, further details and consultation documents can be found at the same site. 